\title{
Acoustically Driven Storage of Light in a Quantum Well
}

\author{
C. Rocke, S. Zimmermann, A. Wixforth, and J. P. Kotthaus \\ Sektion Physik der LMU, Geschwister-Scholl-Platz 1, D-80539 München, Germany \\ G. Böhm and G. Weimann* \\ Walter-Schottky-Institut der TUM, D-85748 Garching, Germany
}

(Received 10 March 1997)

\begin{abstract}
The strong piezoelectric fields accompanying a surface acoustic wave on a semiconductor quantum well structure are employed to dissociate optically generated excitons and efficiently trap the created electron hole pairs in the moving lateral potential superlattice of the sound wave. The resulting spatial separation of the photogenerated ambipolar charges leads to an increase of the radiative lifetime by orders of magnitude as compared to the unperturbed excitons. External and deliberate screening of the lateral piezoelectric fields triggers radiative recombination after very long storage times at a remote location on the sample. [S0031-9007(97)03194-3]
\end{abstract}

PACS numbers: 73.50.Rb, 77.65.Dq, 78.20.Hp, 78.55.Cr

The dynamics of photogenerated carriers in semiconductor structures with reduced dimensionality has been the subject of intensive investigations in recent years $[1,2]$. State-of-the-art band-gap engineering technologies enable us to tailor low-dimensional semiconductor systems with desirable optoelectronic properties and study the fundamental aspects of carrier dynamics. This has increased tremendously our fundamental understanding of the dynamic properties of artificial semiconductor structures and has also resulted in a wide range of novel devices such as quantum well lasers, modulators, and detectors, as well as all-optical switches. Nevertheless, the bulk band structure of semiconductors seems to dominate optoelectronic properties since the strength of interband transitions is largely governed by the atomiclike Bloch parts of the wave function [3]. Thus it appears at first glance unavoidable that strong interband optical transitions are linked to direct band-gap semiconductors with short radiative lifetimes such as GaAs, whereas long radiative lifetimes of photogenerated carriers imply utilization of semiconductors with indirect band gaps such as $\mathrm{Si}$ and correspondingly reduced interband absorption. Initial attempts to employ band-gap engineering in order to combine strong interband absorption with long radiative lifetimes have focused on so-called doping superlattices [4]. There, alternate $n$ and $p$ doping along the growth direction is utilized to combine a direct gap in momentum space with an indirect gap in real space which causes a spatial separation of photogenerated electron-hole $(e-h)$ pairs and hence considerably prolonged lifetimes.

Here, we introduce a new way of band-gap engineering in which we expose a semiconductor quantum well of a direct gap material to a moving potential superlattice modulated in the plane of the well. We show that the confinement of photogenerated $e-h$ pairs to two dimensions, together with the moving lateral superlattice, allows reversible charge separation [5]. We demonstrate that the combination of both the advantages of strong interband absorption and extremely long lifetimes of the optical excitations is achieved without affecting the superior optical quality of the quantum well material.

The spatial separation of the electron-hole pairs is achieved via the piezoelectric potential of acoustic waves propagating along the surface of a semiconductor quantum well system. On a piezoelectric substrate, the elliptically polarized surface acoustic waves (SAWs) are accompanied by both lateral and vertical piezoelectric fields which propagate at the speed of sound. Those fields can be strong enough to field ionize optically generated excitons and to confine the resulting electrons and holes in the moving lateral potential wells separated by one-half wavelength of the SAW. The spatial separation dramatically reduces the recombination probability and increases the radiative lifetime by several orders of magnitude as compared to the unperturbed case. We further demonstrate that the dynamically trapped electron-hole pairs can be transported over macroscopic distances at the speed of sound and that deliberate screening of the lateral piezoelectric fields of the SAW leads to an induced radiative recombination after long storage times at a location remote from the one of $e-h$ generation. This conversion of photons into a long lived $e-h$ polarization which is efficiently reconverted into photons can serve as an optical delay line operating at sound velocities.

The undoped quantum well samples used in our experiments are grown by molecular beam epitaxy on a (100)GaAs substrate. The quantum well consists of $10 \mathrm{~nm}$ pseudomorphic $\mathrm{In}_{0.15} \mathrm{Ga}_{0.85} \mathrm{As}$ grown on a $1 \mu \mathrm{m}$ thick GaAs buffer and is covered by a $20 \mathrm{~nm}$ thick GaAs cap layer. The active area of the sample is etched into a $2.5 \mathrm{~mm}$ long and $0.3 \mathrm{~mm}$ wide mesa (see inset of Fig. 1) with two interdigital transducers (IDTs) at its ends. The IDTs are designed to operate at a center frequency $f_{\text {SAW }}=840 \mathrm{MHz}$. They are partially impedance matched to the $50 \Omega$ radio frequency (rf) circuitry using an on-chip matching network, thus reducing the insertion 


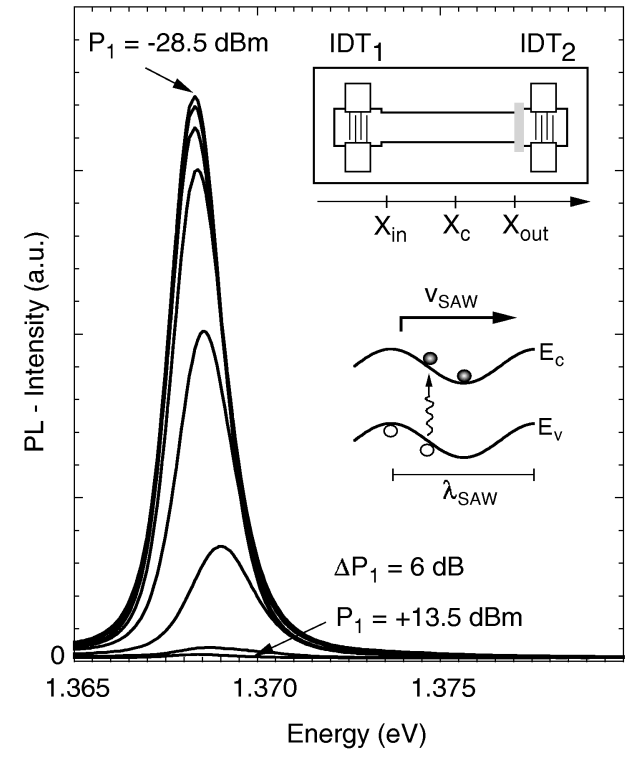

FIG. 1. Photoluminescence spectra of a single $10 \mathrm{~nm}$ wide InGaAs/GaAs quantum well structure for different acoustic powers. The optical excitation occurs at the site $x=x_{c}$ with an intensity of $10 \mathrm{~mW} / \mathrm{cm}^{2}$ and wavelength $\lambda_{\text {laser }}=780 \mathrm{~nm}$. The insets schematically depict the sample design with two interdigital transducers and the storage of optically generated excitons in the potential of a surface acoustic wave.

loss of each transducer to the $5 \mathrm{~dB}$ range. The sample is mounted in an optical cryostat, and the experiments presented here are performed at $T=4.2 \mathrm{~K}$. Light from a pulsed laser diode $\left(\lambda_{\text {laser }}=780 \mathrm{~nm}\right)$ is used for optical interband excitation above band gap, and the photoluminescence (PL) of the sample is analyzed in a triple grating spectrometer. Either a gated photomultiplier or a charged coupled device serve as a detector for the PL. Application of a high frequency signal to one of the IDT launches a SAW of wavelength $\lambda_{\mathrm{SAW}}=v_{\mathrm{SAW}} / f_{\mathrm{SAW}}$. It propagates along the [110] direction and can be detected at the other IDT after the acoustic delay of order of $1 \mu \mathrm{s}$ determined by the spacing of the IDTs. Here, $v_{\mathrm{SAW}}=2865 \mathrm{~m} / \mathrm{s} \mathrm{de}$ notes the SAW velocity for the given sample cut and orientation. Either pulsed or continuous wave (cw) operation of the SAW transducers is possible.

In Fig. 1 we depict the "direct," i.e., local and instantaneous PL of the quantum well under the influence of a SAW. Laser excitation and PL emission occur at the same site $x_{c}$ on the sample close to the center of the mesa with the recombination time being much shorter than the time resolution of our experiment (20 ns). The different PL traces are recorded at different acoustic power levels $P_{1}$ of a cw high frequency $\left(f_{\mathrm{SAW}}=840 \mathrm{MHz}\right)$ signal fed into one of the transducers $\left(\mathrm{IDT}_{1}\right)$. With increasing SAW power the PL is shifted towards slightly higher energies due to the influence of the acoustically induced lateral electric fields via the quantum confined Franz-Keldysh effect [6]. The PL intensity strongly decreases until, at the highest power used, it becomes completely quenched.
This quenching is already an indirect indication of the increased trapping probability and subsequent transport of the $e-h$ pairs, as will be demonstrated in the following.

The piezoelectric fields of the SAW modulate the band edges with respect to the chemical potential similar to doping superlattices [4] or statically imposed laterally periodic electric fields using an interdigitated gate electrode [6]. In this moving potential superlattice with period $\lambda_{\mathrm{SAW}}=3.4 \mu \mathrm{m}$ the excitons become polarized predominantly by the lateral electric field until they dissociate at high fields into spatially separated $e-h$ pairs. These are then efficiently stored in the potential minima and maxima of the conduction and the valence band, respectively (see inset of Fig. 1). For an acoustic power of $P_{1}=+13.5 \mathrm{dBm}(22.4 \mathrm{~mW})$, lateral fields as high as $E_{l}=8 \mathrm{kV} / \mathrm{cm}$ and vertical fields up to $E_{v}=10 \mathrm{kV} / \mathrm{cm}$ are achieved [7-9]. Recent related studies of the optical properties, using statically imposed lateral electric fields on the same wafer [6] and our dynamical experiments, indicate the analogy between both methods to create electric fields in the plane of the quantum well. The influence of the SAW-induced vertical fields $E_{v}$ via the quantum confined Stark effect can be neglected for the given field strengths of $E_{v} \leq 10 \mathrm{kV} / \mathrm{cm}$ since we use a comparatively thin $(10 \mathrm{~nm})$ quantum well $[2,10]$.

The dramatically prolonged recombination time of the trapped $e-h$ pairs, together with the propagation of the SAW along the surface of the quantum well sample, enables us to directly study the transport of the photogenerated carriers over macroscopic distances, as sketched in the inset of Fig. 2. Deliberate screening of the storing lateral potential triggers radiative recombination and thus the reassembly of the polarized $e-h$ pairs into photons. In this experiment, optical excitation and emission of the PL do not occur at the same spot of the sample. Using a pulsed laser diode, the optical excitation is accomplished at the site $x=x_{\mathrm{in}}$, whereas the detector monitors the PL coming from a location $x=x_{\text {out }}$. The part of the sample beyond $x=x_{\text {out }}$ is covered by a thin, semitransparent nickel-chromium layer that readily screens the lateral piezoelectric fields of the SAW $[11,12]$ and thus triggers recombination. At the time $t=0$, a SAW pulse of width $\Delta t=200 \mathrm{~ns}$ is launched at the emitting $\mathrm{IDT}_{1}$. At about $t=t_{1}$, the SAW pulse is centered at $x=x_{\text {in }}$, where a laser pulse creates excitonic excitations. These excitons are immediately field ionized, and the resulting electron-hole pairs are efficiently trapped in the moving lateral potential wells of the SAW, as seen indirectly in Fig. 1. After an acoustic delay time of, in this case, $\tau_{\text {storage }}=350 \mathrm{~ns}$ corresponding to a transport path length of $x_{\text {out }}-x_{\text {in }}=1 \mathrm{~mm}$, the SAW pulse reaches the location $x=x_{\text {out }}$, where the lateral SAW fields are screened, thus inducing recombination and a strong PL signal at the detector photomultiplier. Time delays $\tau_{\text {storage }}$ up to several $\mu$ s corresponding to an $e-h$ transport over some millimeters have been achieved 


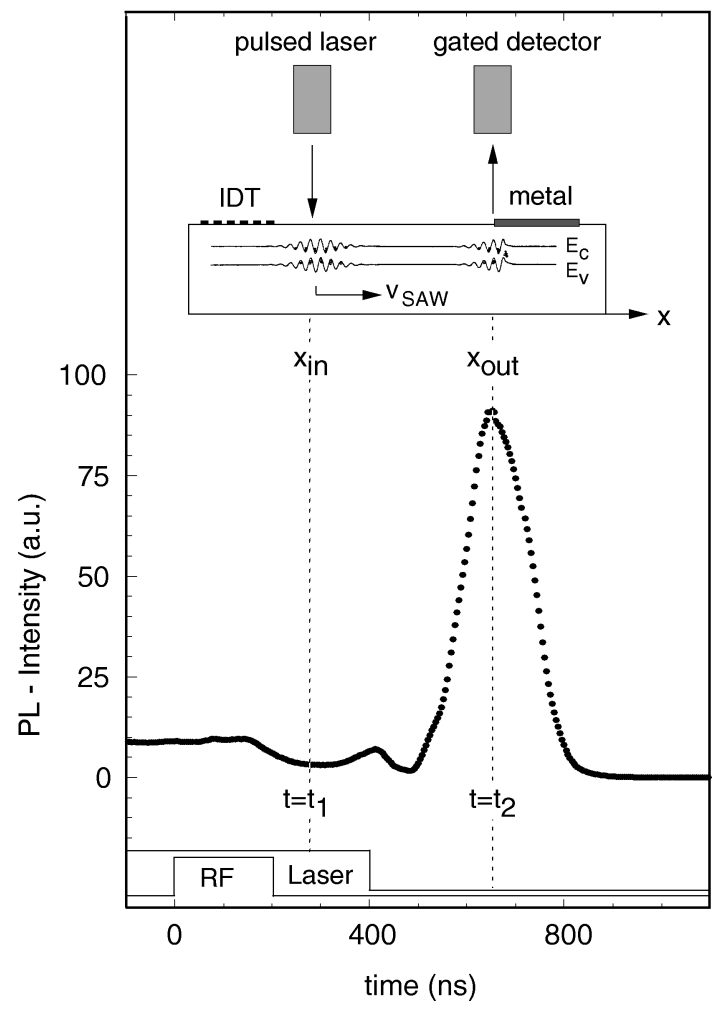

FIG. 2. Ambipolar transport of trapped charges by a SAW. At $t=0 \mathrm{~ns}$ a $200 \mathrm{~ns}$ long rf pulse at $f_{\mathrm{SAW}}=840 \mathrm{MHz}$ applied to $\mathrm{IDT}_{1}$ generates a SAW packet with an acoustic power of $P_{1}=13.5 \mathrm{dBm}$. At $t=t_{1}$ and $x=x_{\text {in }}$ the potential extrema of the SAW are filled with photogenerated electron-hole pairs which are transported with sound velocity to a semitransparent metallization at $x=x_{\text {out }}$. Here the deliberate screening of the piezoelectric potential modulation lifts the spatial separation of the carriers and induces radiative recombination at $x=x_{\text {out }}$ and $t=t_{2}$. The duration of the rf pulse and the laser pulse are indicated in the lower part.

without severe degradation of the transport efficiency, only the sample length imposing a maximum time delay.

The time-delayed PL is demonstrated in the lower part of Fig. 2. Here, we plot the PL intensity detected at $x=x_{\text {out }}$ as a function of time after the SAW excitation. The SAW and the pump laser pulses are indicated by the rectangles close to the ordinate. The slight decrease of the PL intensity at $t=t_{1}$ marks the arrival of the SAW pulse at the excitation site $x=x_{\text {in }}$ by quenching the direct and instantaneous PL at $x=x_{\text {in }}$ that is detected as weak stray light by the photomultiplier. To avoid any possible spurious effect originating from this stray light, we make sure that the pump laser is switched off well before the SAW packet arrives at $x=x_{\text {out }}$. About $650 \mathrm{~ns}$ after the launch of the SAW pulse, we detect a strong PL at the site $x=x_{\text {out }}$ which clearly indicates the SAWmediated transport and subsequent recombination of the photogenerated carriers. Comparison of the PL that is detected at the pumping site $x_{\text {in }}$ to the delayed PL at $x_{\text {out }}$ shows that only about $30 \%$ of the photogenerated and trapped carriers are "lost" along their way. The major mechanisms for this loss are believed to be nonradiative processes along the sound path which might be related to the high density of recombination centers at the mesa edges and to wave front inhomogeneities.

In Fig. 3, we plot the intensity of the delayed PL ( $x=x_{\text {out }}, \tau>300 \mathrm{~ns}$ ) as a function of the SAW power. For comparison, we also show the quenching of the direct and instantaneous PL $\left(x=x_{\mathrm{in}}, \tau<1 \mathrm{~ns}\right)$ as already demonstrated in Fig. 1. Clearly, a threshold behavior for the effective dissociation and transport is observed. Only for SAW powers $P_{1}>-5 \mathrm{dBm}$ carriers are trapped by the SAW and transported to the gate electrode at which they recombine. At the same power level, the direct PL becomes quenched. The corresponding lateral electric field strength of $E_{l} \approx 2 \times 10^{3} \mathrm{~V} / \mathrm{cm}$ agrees very well to the one expected theoretically for exciton dissociation [2]. At high power levels we observe a saturation of the delayed PL signal, which indicates the complete filling of the lateral SAW potential wells and a dynamical screening of the SAW fields by the photogenerated carriers.

The photon reassembly via the recombination of the stored carriers may also be induced following an alternative route, where both the lifetime of the $e-h$ pairs as well as the location of the induced radiative recombination may be chosen at will. In addition to the storing $\mathrm{SAW}_{1}$, a second $\mathrm{SAW}_{2}$ is launched using a second transducer $\mathrm{IDT}_{2}$ (see Fig. 1). When colliding, the two SAWs interfere and create a lateral potential modulation which is oscillating in time and space. If both SAWs have the same wavelength and amplitude, the interference pattern is that of a standing wave. This experiment is depicted in Fig. 4. The acoustic power generated with $\mathrm{IDT}_{1}$ is set to $P_{1}=$ $+4.5 \mathrm{dBm}$, which corresponds to the level at which the

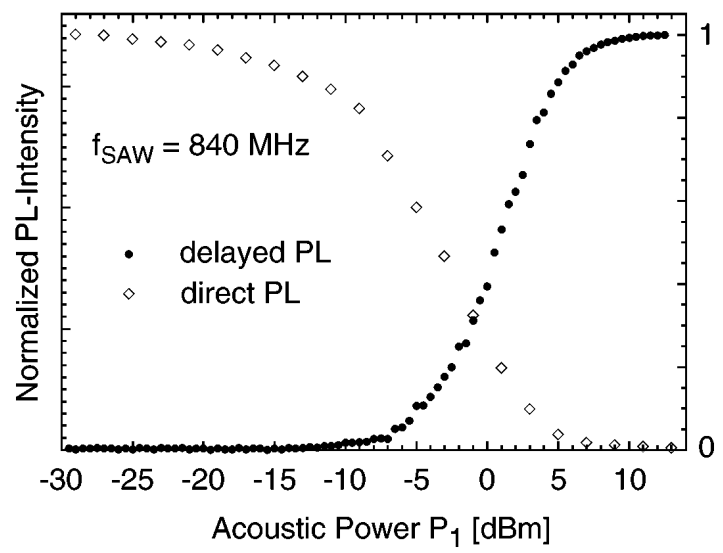

FIG. 3. Normalized direct and delayed PL intensities as a function of the acoustic power $P_{1}$. Efficient ambipolar transport of charges resulting in a time-delayed PL signal requires a minimum threshold acoustic power of $P_{1}=-5 \mathrm{dBm}$ which corresponds to a minimum lateral electric field strength $\left(E_{l}=\right.$ $2 \mathrm{kV} / \mathrm{cm}$ ). This threshold coincides with the quenching of the direct PL signal. 


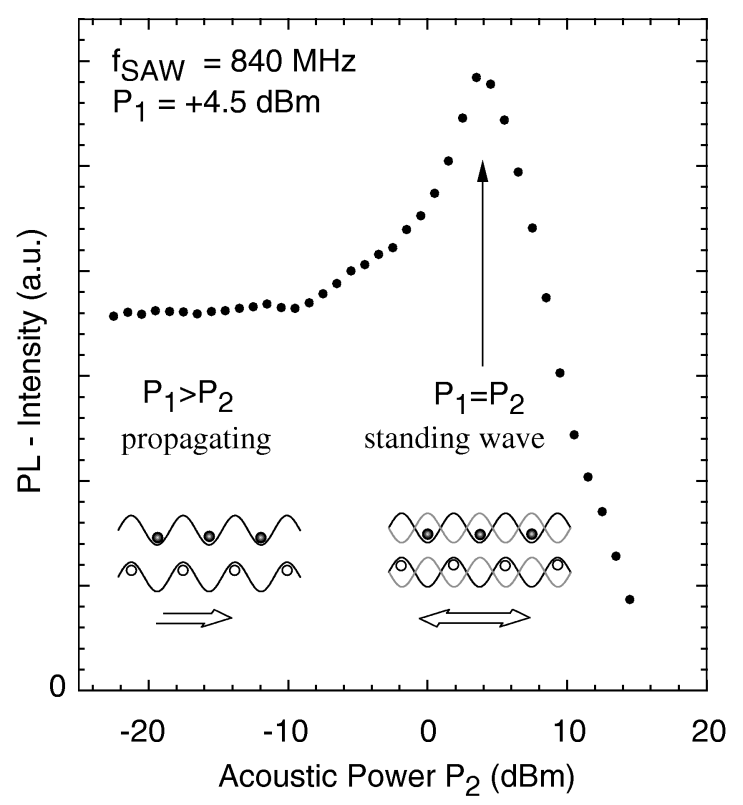

FIG. 4. Direct PL intensity at $x=x_{c}$ as a function of the acoustic power $P_{2}$ for a constant acoustic power $P_{1}=$ $4.5 \mathrm{dBm}$. For the standing wave geometry at $P_{1}=P_{2}$ the spatial separation between electrons and holes is lifted, resulting in a recovered PL intensity due to the increased transition probability.

direct PL is already quenched by about $50 \%$ (cf. Figs. 1 and 3). The power level $P_{2}$ of the counterpropagating $\mathrm{SAW}_{2}$ of the same frequency $f_{\mathrm{SAW}}=840 \mathrm{MHz}$ is varied in the experiment. As long as $P_{1}>P_{2}, \mathrm{SAW}_{1}$ is effectively trapping and transporting the photogenerated $e-h$ pairs. However, if $P_{2}$ becomes comparable or equal to $P_{1}$, both waves interfere to create a standing wave pattern. At this moment, the time averaged wave function overlap of the stored electrons and holes increases dramatically, which results in an induced radiative recombination and a strong increase of the observed PL. This result clearly excludes any possible thermal effect that might be responsible for the quenching of the PL. Further increase of $P_{2}$ reverses the original situation, as now $\mathrm{SAW}_{2}$ takes over the charges and efficiently reduces the recombination probability again by a spatial separation of the $e-h$ pairs. This is clearly demonstrated by the strong decrease of the PL intensity at high power levels $P_{2}>P_{1}$.

In summary, we demonstrate the transformation of light into an elementary excitation of a solid stored in the lateral superlattice potential of a surface acoustic wave moving at the speed of sound. The corresponding storage time can be orders of magnitudes longer than the recombination lifetime in direct band-gap semiconductors. Reassembly of the stored charges into photons can be in- duced at a location distant from their generation. This way, the lifetimes of the optical excitations in a direct semiconductor can be prolonged by several orders of magnitude without degradation of the superior optical properties of the high purity material. The possibility of tuning the storage time of the light-generated $e-h$ polarization by two counterpropagating SAWs, together with the fact that the location of the radiative recombination on the sample can be determined only by a preset time delay between the two SAW pulses, opens a wide field for novel acoustooptic devices. Optical delay, beam steering, multiplexing, and demultiplexing of optical signals possibly may thus be realized on a single chip.

We gratefully acknowledge very enlightening discussions with A.V. Govorov, technical advice from $\mathrm{S}$. Manus, and the financial support of the Deutsche Forschungsgemeinschaft (DFG) and the Bayerische Forschungsstiftung FOROPTO.

*Now at Fraunhofer Institut IAF, D-79108 Freiburg, Germany.

[1] S. Schmitt-Rink, D. S. Chemla, and D. A. B. Miller, Adv. Phys. 38, 89 (1989).

[2] D. A. B. Miller, D. S. Chemla, T. C. Damen, A. C. Gossard, W. Wiegmann, T. H. Wood, and C. A. Burrus, Phys. Rev. B 32, 1043 (1985).

[3] See, e.g., C. Weisbuch and B. Vinter, Quantum Semiconductor Structures (Academic Press, San Diego, 1991).

[4] G. H. Döhler, H. Künzel, D. Olego, K. Ploog, P. Ruden, and H. J. Stolz, Phys. Rev. Lett. 47, 864 (1981); R. A. Street, G. H. Döhler, J. N. Miller, and P.P. Ruden, Phys. Rev B 33, 7043 (1986).

[5] Transport of unipolar charges in epitaxial films is usually referred to as acoustic charge transport (ACT). See e.g., M. J. Hoskins and B. J. Hunsinger, Appl. Phys. Lett. 41, 332 (1982); W. J. Tanski, S. W. Merritt, R. N. Sacks, D. E. Cullen, E. J. Branciforte, R. D. Carroll, and T. C. Eschrich, Appl. Phys. Lett. 52, 18 (1988).

[6] A. Schmeller, W. Hansen, J. P. Kotthaus, G. Tränkle, and G. Weimann, Appl. Phys. Lett. 64, 330 (1994).

[7] Y. Kim and W. D. Hunt, J. Appl. Phys. 68, 4993 (1990).

[8] J. F. Jain and K. K. Bhattacharjee, IEEE Photonics Technol. Lett. 1, 307 (1989).

[9] T. Gryba and J. E. Lefebvre, IEE Proc., Optoelectron. 141, 62 (1994).

[10] L. Vina, E. E. Mendez, W. I. Wang, L. L. Chang, and L. Esaki, J. Phys. C 20, 2803 (1987).

[11] A. Wixforth, J. Scriba, M. Wassermeier, J.P. Kotthaus, G. Weimann, and W. Schlapp, Phys. Rev. B 40, 7874 (1989).

[12] C. Rocke, S. Manus, A. Wixforth, M. Sundaram, J. H. English, and A.C. Gossard, Appl. Phys. Lett. 65, 2422 (1994). 